\title{
Regulation of Stem Cells in Their Niche
}

\author{
Li Ming Gooi ${ }^{1}$ - Jay Gopalakrishnan ${ }^{1}$
}

Published online: 31 May 2016

(C) Springer International Publishing AG 2016

\begin{abstract}
Stem cells are characterized by their ability to asymmetrically divide, generating another self-renewing stem cell and a differentiating daughter cell. They reside within the local niche microenvironment, which, together with systemic signals, regulates intrinsic stem cell function. Stem cells are finely regulated, with even slight deregulation leading to gain of stem cell function and tumorigenesis or loss of stem cell function and tissue degeneration in aging. In this review, we highlight how stem cells are maintained within their niche and what is known about their deregulation in aging. To highlight these points, we look at the Drosophila male germline stem cell niche as a model system, specifically focusing on cell cycle progression, signaling pathways, epigenetics, and the control of spindle orientation by centrosomes. Finally, we sum up pertinent questions to be addressed in understanding stem cell function and their malfunction in aging.
\end{abstract}

Keywords Stem cells $\cdot$ Drosophila male germline $\cdot$ Cell cycle $\cdot$ Centrosomes $\cdot$ Aging $\cdot$ Epigenetics $\cdot$ Signaling

\section{Introduction}

Stem cells are characterized by two properties, namely their ability to self-renew and their potency in forming other cell

This article is part of the Topical Collection on Age-related Stem Cell Modifiers

Jay Gopalakrishnan

jay.gopalakrishnan@uni-koeln.de

1 Laboratory for Centrosome and Cytoskeleton Biology, Institute for Biochemistry I, and Center for Molecular Medicine of the University of Cologne, Robert-Koch-Str. 21, 50931 Cologne, Germany types, which is achieved through the process of asymmetric division where a stem cell divides to produce another selfrenewing stem cell and a daughter cell that is one step closer to differentiation. The number of steps required prior to terminal differentiation varies for different tissues [1]. Stem cells are first encountered in the embryo where totipotent stem cells that can divide to form all cell types of an organism give rise to a fully functioning adult.

In adults, certain tissues and organs retain unipotent stem cells that only differentiate to form mature cells of one lineage. They thus partake in homeostatic cell replacement and tissue regeneration, maintaining organ function throughout an organism's lifetime. As an organism ages, tissues and organs start to deteriorate. One of the postulated hallmarks of aging is stem cell exhaustion, which can include both the loss of stem cells from their niche, as well as a decline in their regenerative potential, and is usually the combined consequence of different factors [2]. However, the underlying mechanisms leading to this loss of stem cells remain poorly understood.

How stem cells are regulated and the changes in aging remain unclear. Stem cells reside within a microenvironment known as the niche. The stem cell niche provides extrinsic extracellular cues, which then initiate intrinsic changes to control cellular dynamics through which stem cells are maintained in their undifferentiated and self-renewable state [3]. As a stem cell leaves its niche, either due to the axis of division, loss of adhesion or space constraints, it is then exposed to differentiation signals, altering internal genetic programs, which guide it to form particular cell lineages. Thus, dissecting the intrinsic, extrinsic, and systemic factors that regulate stem cells and their alterations during aging requires a genetically tractable model system [4].

The fruit fly Drosophila melanogaster is a well-established model organism with biological mechanisms and pathways that are conserved with mammals. Research in recent years 
has identified various stem cell systems in developing as well as adult flies. One of these is the male germline stem cell (GSC) niche, which is quite well described, thus making it suitable for the study of stem cells, the factors that maintain them and their deregulation in aging.

\section{Establishment and Structure of the Stem Cell Niche}

Fertilization between the ovum and sperm results in a totipotent zygote, which develops into a complete organism. During development, various stem cell niches are established, providing anatomical and functional spaces for adult stem cells. Stem cell establishment usually precedes or coincides with niche formation and involves a series of actions through which non-selfrenewing precursor cells acquire stem cell characteristics.

The establishment of the male GSC niche in Drosophila starts at stage 13 to 14 of embryogenesis, when primordial germ cells (PGCs) and somatic gonadal precursors (SGPs) come together to form the testis. Two populations of SGPs exist, Abdominal-B-specified male specific SGPs (msSGPs) at the posterior end of the testis and escargot expressing SGPs at the anterior end [5]. It is the escargot expressing SGPs that then give rise to the embryonic hub. At stage 13 of embryogenesis, all germ cells throughout the testis display JAK-STAT (janus kinase-signal transducer and activator of transcription) activation. However, at stage 17, JAK-STAT activation is restricted to the germ cells at the anterior end of the testis where hub cells secreting the JAK-STAT ligand, Upd, reside [5]. Studies have shown that JAK-STAT signaling is essential for conferring stemness to the PGCs [5]. By the middle of stage 17 embryos, these initial GSCs are already observed to divide with a plane perpendicular to the hub, typically characteristic of adult male GSCs.

As mentioned above, at the tip of the testis lies a cluster of 10 to 18 somatic post-mitotic cells forming the hub. In young adults, these hub cells are surrounded by 8 to 15 GSCs, each of which is flanked by a pair of somatic cyst stem cells (CySCs). Asymmetric division of the GSC produces a differentiating daughter gonialblast (GB), which is also surrounded by a pair of cyst cells (CyCs) formed from CySCs. The GB then initiates differentiation, undergoing four rounds of mitotic division with incomplete cytokinesis, resulting in the stepwise formation of 2-, 4-, 8- and 16-cell interconnected spermatogonial cysts (Fig. 1a) [6]. The 16 interconnected spermatogonia then differentiate into spermatocytes, the whole process lasting around 2 days. Spermatocytes enter into an extended G2-meiotic prophase I transition lasting 3.5 days, during which they undergo DNA synthesis, increasing 25fold in volume and initiating an extensive transcription program to prepare for spermatid formation [7]. From metaphase I onwards, spermatocytes rapidly undergo 2 meiotic divisions generating a cyst of 64 haploid spermatids, which mature to form motile sperm [8].

\section{The Cell Cycle in Drosophila Male GSCs}

Although general cell cycle components and progression are well characterized and conserved across many different species and cell types, the exact regulation of cell cycle progression in GSCs still remains to be elucidated. The cell cycle is composed of four successive phases: mitosis (M), gap 1 (G1), synthesis (S), and gap 2 (G2), each characterized by specific events, and culminating in the successful division of a cell (Fig. 1b). At the transition between phases, various switch-like checkpoints exist to ensure that preceding cell cycle events are correctly completed before allowing later events to occur [9].

Cell cycle progression involves the complex interplay of various cyclin-dependent kinases (CDKs) and cyclins. CDKs contain a protein kinase subunit, the activation of which requires binding of a partner cyclin and an activating phosphorylation on a threonine residue in the vicinity of its active site. In the classical model of cell cycle control, specific cyclinCDK combinations facilitate the transition between the different phases (Fig. 1b), with the main events controlled by CDK1, whose activation promotes entry into mitosis, and APC, whose activation drives exit from mitosis [10]. Although the cell cycle network is well characterized, cell type specific differences remain to be discovered.

In addition to cyclins and CDKs, other major regulator proteins include Wee1, Cdc25, and Plk1. Wee1 inactivates cyclin-CDK1 by phosphorylation of a tyrosine residue (Y15) and a threonine residue (T14) to restrain entry into mitosis. On the other hand, the protein phosphatase $\mathrm{Cdc} 25$ dephosphorylates CDK1 on these residues to initiate $\mathrm{G} 2 / \mathrm{M}$ transition and thus entry into mitosis. Both Wee1 and Cdc25 are regulated by signaling pathways controlling G2/M-transition, thus providing a feedback regulatory mechanism to ensure accurate cell cycle progression [9].

There has been an emergence of research linking the cell cycle to stem cell states and fates. Certain stem cells possess a cell cycle uniquely different to that of differentiating cells, leading to the question of how and what role the cell cycle plays in stem cells $[11,12]$. In the Drosophila testis, GSCs divide frequently in an asynchronous manner. Previous studies show them to divide every $12-16 \mathrm{~h}$ with mitosis lasting $\sim 30$ min $[13,14]$ (Fig. 1b). At any given time, $\sim 7 \%$ of GSCs were observed to be in the G2 phase, while $\sim 3-4 \%$ of GSCs were in mitosis [15]. After mitosis, GSCs enter an extremely short G1 phase. Both GSCs and GBs then appear to synchronously enter $\mathrm{S}$ phase without undergoing cytokinesis, with cytokinesis being completed at early G2 [16].

The components of the cell cycle appear to be conserved in the male GSC niche. In mutants of cyclin B, which binds to 
a. Drosophila male testis and Germline stem cells (GSCs) niche

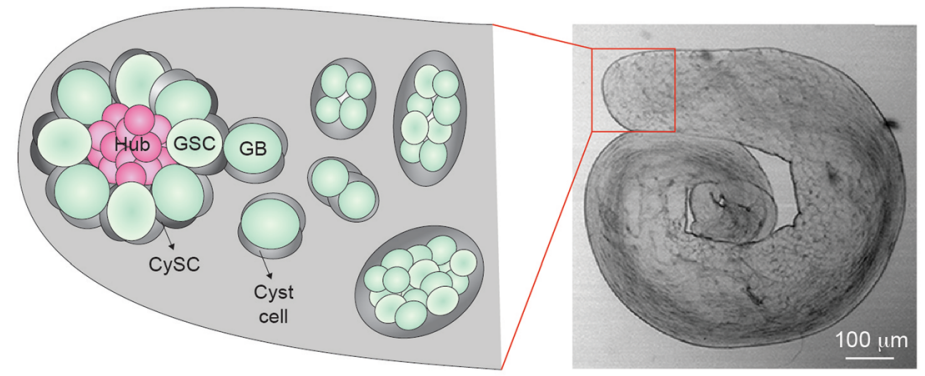

C. Defects in GSCs

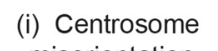
misorientation

(ii) Spindle

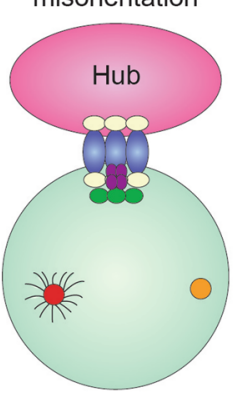

misorientation

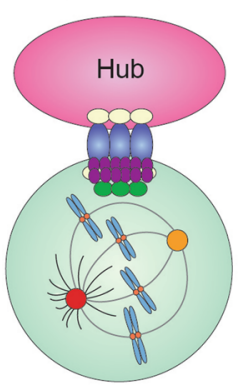

b. Centrosome orientation and the cell cycle
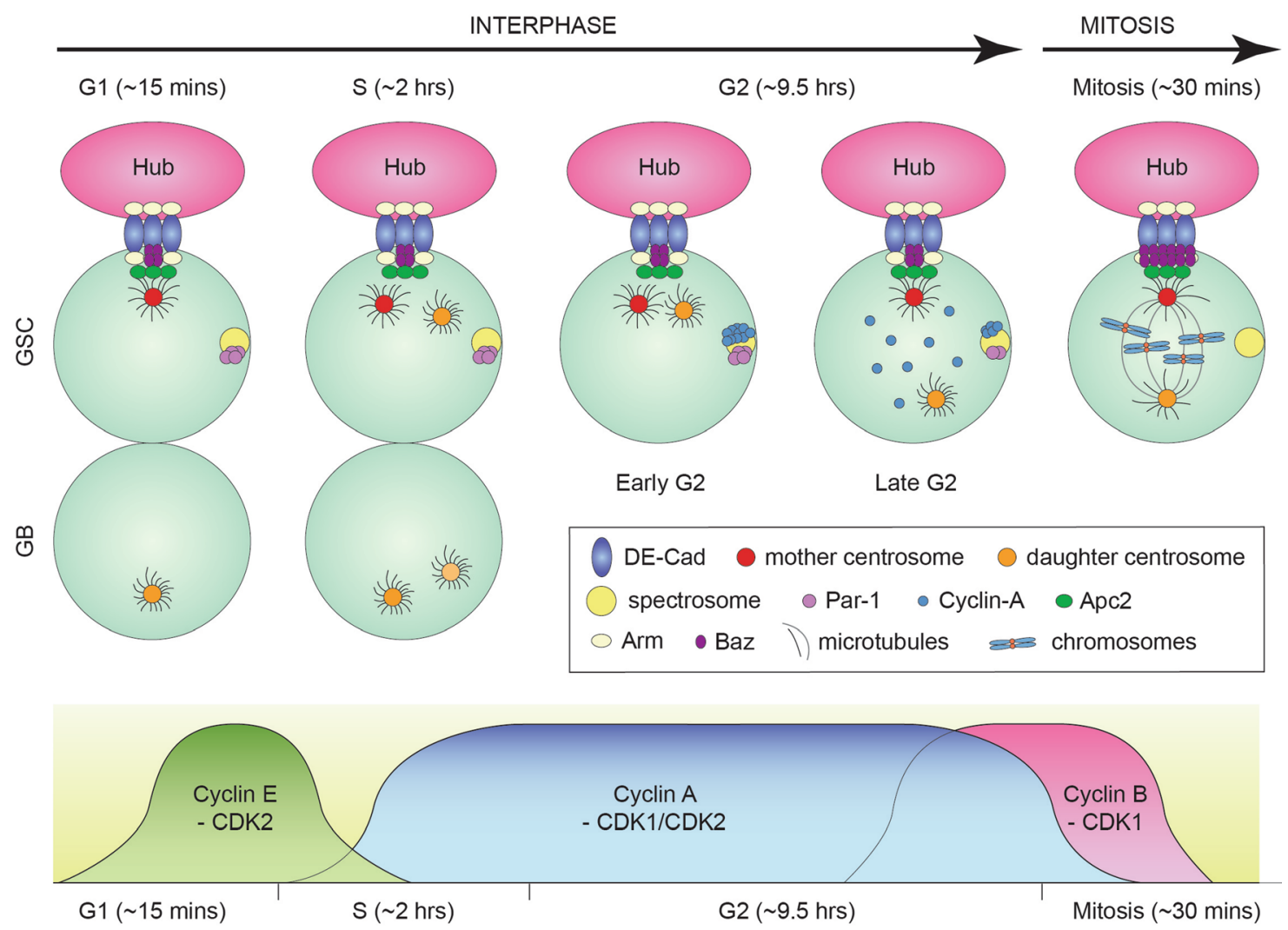

Fig. 1 a A young Drosophila testis with the male germline stem cell niche highlighted by a red box and shown on the left. Germline stem cells (GSCS) surround post-mitotic hub cells to form a rosette structure. These GSCs then asymmetrically divide to form 2-, 4-, 8- and 16-cell interconnected spermatogonial cysts, which undergo meiosis and maturation to form sperm. b Coordination of centrosome orientation in GSCs with cell cycle progression. The mother centrosome remains in the vicinity of the hub-GSC interface, while the daughter centrosome moves away to the hub-distal pole to be segregated into the daughter GB upon cellular division. Various components of the centrosome orientation checkpoint $(C O C)$ and the changes they undergo during the cell cycle are shown. The corresponding changes in cyclin-dependent kinases $(C D K s)$ and cyclins are also shown in the graph at the bottom. Cyclin E-CDK2 initiates S phase. Cyclin A and either CDK1 or 2 then plays a role in the continuation of $\mathrm{S}$ phase and B-type cyclins, together with CDK1 is responsible for entry into mitosis. c (i) Centrosome misorientation where neither centrosome is located in the proximity of the hub-GSC junction. (ii) Centrosome misorientation and inactivation of the $\mathrm{COC}$ results in spindle misorientation during mitosis
CDK1 to initiate entry into mitosis, a reduction and loss of GSCs is observed. However, testicular somatic cells do not seem to require cyclin B as cyst and hub cells appear normal in cyclin B mutants. The observed defects in the GSCs could not be rescued by cyclin A or cyclin B3, thus showing the non- redundant functions of different cyclins [17]. String (Stg), the Drosophila homolog of Cdc25, is highly expressed in GSCs and CySCs and rapidly downregulated in their differentiating daughters (GBs, spermatogonia and cyst cells, CC). It is required in both GSCs and CySCs where increase or decrease in 
Stg activity in each stem cells affects its neighboring stem cell depicting the complex interplay between stem cells in their niches [18].

Together, these studies illustrate the complexity of the cell cycle in different stem cells within their niche, and how the cell cycle of a stem cell influences and controls neighboring stem cells. The male GSC niche thus provides a simplified system to study this in detail.

\section{Centrosomes and the Plane of Cell Division}

Centrosomes regulate mitotic spindle orientation to determine the plane of cell division thus ensuring accurate stem cell division. The Drosophila male GSCs in their niche provide one of the best-characterized systems to study this. In male GSCs, the mitotic spindle is oriented perpendicular to the GSC-hub interface, thus ensuring that one daughter cell stays in contact with the hub to receive sustaining signals and maintain its stemness, while the daughter cell is displaced away from the niche and initiates differentiation. Various checkpoints are in place to halt cell division when the requirements are not met for accurate cell division. One of these checkpoints includes the centrosome orientation checkpoint (COC), which monitors centrosome orientation and functions during the G2$\mathrm{M}$ transition of the cell cycle.

Centrosomes, highly conserved organelles that are the main microtubule organizing centers (MTOCs) of cells, play a role in establishing the stereotypical spindle orientation in GSCs. During G1, a single centrosome is situated at the hubGSC interface [14]. This centrosome duplicates, and by G2, separates. The mother centrosome, which is faithfully retained by GSCs across many cellular divisions, remains in the vicinity of the hub-GSC interface, while the daughter centrosome moves away to the hub-distal pole to be segregated into the daughter GB upon cellular division [19••]. However, mother and daughter centrosomes possess different characteristics. Hub-distal daughter centrosomes have very little associated microtubules during interphase, allowing their free movement around the GSC and developing robust microtubule asters during mitosis. Hub proximal mother centrosomes have robust microtubule arrays even during interphase, with astral microtubules extending towards the adherens junctions, composed of DE-cadherin and Armadillo, which provide a scaffold for the localization of Apc2 (Adenomatous Polyposis Coli 2), thus enabling astral microtubule docking to orient centrosome positioning (Fig. 1b).

The COC in GSCs ensures correct centrosome orientation before mitosis, where in misoriented GSCs, where neither centrosome is located adjacent to the hub, cell cycle progression is delayed until centrosome positioning is re-oriented, thus preventing the formation of misoriented spindles. Various components of the $\mathrm{COC}$ have been described. Among them is the polarity protein Bazooka (Baz), also known as Par-3 [20, 21]. Baz-centrosome association is potentially the key physical event monitored by the $\mathrm{COC}$ in addition to Baz being required for centrosome orientation itself. Baz forms a confined $\sim 1.5 \mu \mathrm{m}$ subcellular structure (Baz-patch) at the hub-GSC interface during interphase. Docking of the apical centrosome at the Bazpatch occurs during late $\mathrm{G} 2$ of the cell cycle, coinciding with Par-1-dependent phosphorylation of Baz at serine 151 (S151). Upon entry into mitosis, Baz localization diffuses, phosphorylation signals become undetectable, and Baz-centrosome docking could no longer be defined. Centrosome-Baz association is thus interpreted by GSCs to indicate "correct centrosome orientation" resulting in the inactivation of the $\mathrm{COC}$ and allowing mitotic progression (Fig. 1b) [16].

Another COC component is the cell polarity gene par-1, which links the COC to cell cycle progression. Par-1 localizes to the spectrosome during interphase, with diminished localization during mitosis. Cyclin $\mathrm{A}$ is barely detectable during the G1/S stage of the cell cycle, while in G2, cyclin A levels increase, initially localizing to the spectrosome, then spreading to the cytoplasm. At prophase to prometaphase, cyclin A is observed both at the nucleus and in the cytoplasm, and by metaphase cyclin A is degraded, signifying the onset of anaphase. In wild type flies, cytoplasmic cyclin A is not observed when centrosomes are misoriented. However, in par-1 mutants, cytoplasmic cyclin A is observed even when centrosomes were misoriented, with a corresponding increase in spindle misorientation. Par-1 thus acts as a gatekeeper of the $\mathrm{COC}$, keeping cyclin A at the spectrosome when centrosomes are misoriented. When centrosomes reach the right orientation, Par-1 releases cyclin A to the cytoplasm and nucleus, allowing entry into mitosis (Fig. 1b) [22].

Apart from the COC, the SAC (spindle assembly checkpoint) also exists in GSCs and monitors the tension at kinetochores generated by microtubule attachments during the metaphase-anaphase transition [23]. Together, these checkpoints ensure that correct centrosome and spindle orientation is achieved to accurately segregate chromosomes and cell fate determinants for asymmetric cell division.

\section{Epigenetic Regulation of Stem Cells}

Epigenetics refer to a wide range of phenomena, which result in a usually heritable change of gene expression without affecting primary DNA sequence. Drosophila male GSCs show a unique segregation of epigenetic cues, where existing H3 histones are distinctly retained by GSCs, while newly synthesized H3s are partitioned into differentiating GBs [24]. This is achieved by phosphorylation of existing $\mathrm{H} 3$ at threonine 3, preceding mitotic entry. During mitosis, phosphorylated H3 is then separated into GSCs, while newly synthesized, unphosphorylated H3 is separated into GBs [25••]. The 
Haspin protein, which also regulates mitotic spindle polarity $[26,27]$, has been shown to be potentially responsible for this phosphorylation [28].

It should be noted that although histones are non-randomly segregated based on age, the genetic material they package does not necessarily share the same fate. While $\mathrm{X}$ and $\mathrm{Y}$ chromosomes are non-randomly segregated between GSCs and GBs, the autosomes (chromosomes 2 and 3) appear to be randomly segregated [29•]. The proteins cnn, KOI, KLAR and Dnmt2 play a role in this segregation pattern, where cnn is an essential centrosomal protein also partaking in the COC, and KOI and KLAR are components of the LINC (linker of nucleoskeleton and cytoskeleton) complex connecting the nucleus to the cytoskeleton [30-32]. Dnmt2 is postulated to facilitate non-random $\mathrm{X}$ and $\mathrm{Y}$ chromosome segregation through DNA sequence independent modifications during gametogenesis in parent flies [33]. It would thus be interesting to elucidate the mechanisms correlating this difference in segregation of histones and sister chromatids.

Another histone modifier, Enhancer of Zeste (E(z)), is also involved in maintaining male germline identity through its involvement in the Polycomb repressive complex 2 (PRC2) [34]. $\mathrm{E}(\mathrm{z})$, a histone methyltransferase, trimethylates lysine 27 of histone $\mathrm{H} 3$ (H3K27me3) [35]. H3K27me3 has then been shown to be enriched at the gene locus of Zinc-finger homeodomain protein 1 (Zfh-1), which marks CySCs and early cyst cells. Interestingly, $\mathrm{E}(\mathrm{z})$ functions in CySCs in a non-cell autonomous manner to convert early stage-germ cells (GSCs, GBs, and/or 2cell spermatogonia) to cyst cells. It has thus been hypothesized that lineage conversion occurs through $\mathrm{E}(\mathrm{z})$-mediated changes to EGFR and Wg signaling between both germ- and cyst-cells, as H3K27me3 is enriched at the gene loci of components of both pathways, with removal of one copy of egfr (EGF receptor) sufficient to suppress $\mathrm{E}(\mathrm{z})$ null phenotypes [36].

Little imaginal discs (lid) is a transcriptional repressor that demethylates $\mathrm{H} 3 \mathrm{k} 4 \mathrm{me} 3$, which is generally associated with transcriptionally active genes [37, 38]. In male GSCs, lid cell - autonomously regulates JAK-STAT activity through the control of Stat92E transcript levels to maintain GSC number and mitotic index and prevent premature GSC differentiation [39]. While lid is required for GSC maintenance, ubiquitously transcribed tetratricopeptide repeat gene on the $\mathrm{X}$ chromosome (dUTX) regulates the proper behavior of CySCs and early cyst cells, preventing them from overpopulating the niche, thus maintaining the proper architecture of the niche. dUTX regulation of cyst cells is exerted through its demethylase activity that abolishes trimethylation on lysine 27 of histone H3 (H3K27me3) at the gene locus of Socs36E, which suppresses JAK-STAT signaling [40].

Undoubtedly, epigenetics play important roles in the Drosophila male GSC niche. However, much work is still needed to clarify how they regulate and maintain the different stem cells in the GSC niche.

\section{Signaling Pathways in the Drosophila Male GSC Niche}

Signaling pathways are activated through binding of specific ligands to transmembrane receptors that initiate intracellular signaling cascades, resulting in the up- or downregulation of target genes, thus controlling protein levels and activation to alter cellular dynamics and determine cell fate and activity.

The JAK-STAT pathway is well characterized in the Drosophila male GSC niche with pathway components shown to be involved in regulating cell-cycle progression [41]. JAK-STAT pathway activation requires binding of its secreted ligand Unpaired (either Upd1, Upd2 or Upd3) to its transmembrane receptor Domeless (Dome), which undergoes a conformational change, allowing the phosphorylation of the intracellular JAK kinase homolog Hopscotch (Hop). This allows the binding of the cytoplasmic STAT homolog Stat92E to the activated Dome-Hop complex, leading to phosphorylation of Stat92E, which dimerizes and translocates to the nucleus to control target gene expression [42].

Hub cells produce and secrete glycosylated Upd, thus restricting its diffusion from the hub [43]. This is crucial in the accurate maintenance and self-renewal of both GSCs and CySCs. Loss of JAK-STAT activity in GSCs and CySCs lead to their loss from the hub, while ectopic expression of Upd was sufficient to increase the number of GSC- and CySC-like cells. JAK-STAT activity is differentially regulated in the three cell types of the niche. CySCs express higher levels of the JAK-STAT signaling inhibitor, suppressor of cytokine singling 36E (SOCS36E) in comparison to GSCs [44]. High SOCS36E expression levels are also observed in the hub cells. Thus, although Upd secreted from hub cells binds to the JAKSTAT receptor Dome in all cell types of the niche, higher levels of JAK-STAT pathway activation are observed in GSCs. This is required for ensuring a proper ratio of the GSC and CySC lineages in the niche [45]. In GSCs, JAKSTAT signaling mediates hub-GSC adhesion via DE-cadherin, and reduced pathway activation has been shown to result in mislocalization of DE-cadherin and GSC loss from the hub.

In addition to JAK-STAT signaling, BMP signaling also plays an essential role in preventing GSCs from differentiating $[46,47]$. This is achieved through its ligands Gbb (Glass bottom boat) and Dpp (Decapentaplegic), which are secreted from hub cells and CySCs to initiate BMP signaling in GSCs. BMP signaling in GSCs represses expression of the differentiation factor bam thus maintaining GSCs and their ability to self-renew [48]. Interestingly, recently identified nanotubes, which are microtubule-based structures, were shown to specifically play a role in BMP signaling in GSCs. Nanotubes extend into the hub, possibly allowing GSCs to selectively access the high threshold level of BMP ligand necessary for self-renewal [49•]. 
Other signaling pathways are also involved in regulation of the Drosophila male GSC niche, including the Insulin-, TOR (target of Rapamycin)-, EGFR (epidermal growth factor receptor)-, MAPK (mitogen-activated protein kinase)-, and Hh (Hedgehog)-signaling pathways [50-53]. However, how these pathways interact with each other to regulate male GSCs is beyond the scope of this review.

\section{Deregulation of the Drosophila Male GSC Niche in Aging}

How the young Drosophila male GSC niche is maintained is well characterized. Recent studies have started elucidating the changes observed in this system upon aging, thus providing a firm foundation on which to further explore how stem cells age in vivo.

The male GSC niche undergoes progressive aging leading to a gradual decrease in overall function. In the initial stages of aging, germ cell numbers decrease, resulting in the shrinkage of overall testis size. There is however no drastic change in GSC numbers. Instead, this decline is caused by a decrease in the rate of GSC asymmetric divisions, due to a reduction in cell cycle activators and increased centrosome misorientation [13].

Stg is the Drosophila homolog of Cdc25, a phosphatase that activates CDKs. During initial aging (day 20), the levels of Stg decrease in GSCs. Surprisingly, this decrease in Stg is not observed in neighboring CySCs, which highlights the differences in stem cell maintenance, even within the same niche. GSCs exhibit decreased Stg and cell division while maintaining its numbers, whereas CySCs decrease in numbers yet show no decrease in Stg levels or division rate. It should also be noted that at this stage of aging, the decrease in Stg levels did not lead to centrosome misorientation [18].

Decreased GSC division rates are also caused by centrosome misorientation, which increases to its maximum by day 30. This level of centrosome misorientation is observed even in 50-day-old flies. Centrosome misorientation is defined as the condition where neither centrosome is situated next to the hub. Misoriented centrosomes then lead to arrest of GSCs or a transient cell cycle delay. GSCs with misoriented centrosomes rarely overcome the $\mathrm{G} 1 / \mathrm{S}$ transition to progress on to mitosis, and thus misoriented spindles are rarely observed. Concurrently, misoriented GSCs divide less frequently. However, upon correction of centrosome orientation, GSCs re-enter cell cycle. Research has shown misoriented GSCs to arise from dedifferentiated spermatogonia (Fig. 1c) [13, 54].

Aging also leads to a decrease in Upd expression by hub cells, resulting in a loss of GSCs from its niche. This is due to the decrease in levels of the IGF-II messenger RNA binding protein (Imp), which functions by preventing endogenous siRNAs present in hub cells from degrading upd RNAs. The decrease in Imp is caused by an increase in let-7 miRNA during aging which destabilizes Imp through seed sequences on Imp's 3'UTR. Constitutive expression of upd only in hub cells could restore the GSC numbers in aging. However, these GSCs showed reduced proliferation, lower than that of aged controls, and the flies displayed decreased lifespan [55].

Despite the wealth of studies on epigenetics in aging as a whole, epigenetic changes in the male GSC niche during aging are not well studied. However, the epigenetic regulators of the male GSC niche do play a role in lifespan at the whole organism level. Mutations in E(z) lead to an increased lifespan in flies [56]. Male Lid mutants possess shortened lifespans [57]. In fact, overexpression of RBR-2, the orthologue of Drosophila Lid, in the germline of $C$. elegans led to an increased lifespan, giving rise to the notion that $\mathrm{H} 3 \mathrm{k} 4 \mathrm{me} 3$ demethylation in the germline can maintain somatic cells [58]. Overexpression of histones $\mathrm{H} 3$ and $\mathrm{H} 4$ extends the replicative lifespan of yeast, leading to the question of whether asymmetric segregation of old and new $\mathrm{H} 3$ in the male GSC niche are affected in aging [59].

The above-mentioned aspects highlight changes observed in the aging male GSC niche. However, many questions still remain unanswered. Although increased centrosome misorientation in aging leads to a delayed cell cycle, it is not well elucidated how and if that is the only cause leading to delays in cell cycle progression. In addition, despite JAK-STAT signaling having been shown to play a role in the cell cycle, it is also not well elucidated if and how this is connected to centrosome misorientation and delayed cell cycle progression. Finally, what are the epigenetic changes observed in the male GSC niche? Further studies are thus warranted to identify if these changes observed in aging are causative or rather just coincidental, and how they interact with and influence each other, leading to aging.

\section{Conclusions}

Recent years have seen a huge interest in stem cell research and its potential therapeutic possibilities [60]. Despite what is already known, much remains to be studied about how systemic, extrinsic, and intrinsic factors coordinate to regulate stem cell function. The fruit fly Drosophila melanogaster provides a simplified model organism to study these in vivo, providing fundamental concepts which can be further extrapolated to accelerate research in mammalian systems. This will be greatly helpful in facilitating medical applications of stem cells by improving safety, efficacy, and cost, with the end aim of revolutionizing medicine to benefit mankind.

Acknowledgments The authors apologize to those whose work were not cited due to space constraints. We thank members of the Gopalakrishnan lab for helpful discussions and comments on the manuscript. This work was supported by Deutsche Forschungsgemeinschaft (DFG) Grant GO 2301/2-1. 


\section{Compliance with Ethical Standards}

Conflict of Interest Li Ming Gooi and Jay Gopalakrishnan declare that they have no conflict of interest.

Human and Animal Rights and Informed Consent This article does not contain any studies with human or animal subjects performed by any of the authors.

\section{References}

Papers of particular interest, published recently, have been highlighted as:

- Of importance

•- Of major importance

1. Roubinet C, Cabernard C. Control of asymmetric cell division. Curr Opin Cell Biol. 2014;31:84-91. Review.

2. López-Otín C, Blasco MA, Partridge L, et al. The hallmarks of aging. Cell. 2013;153(6):1194-217. Review.

3. Scadden DT. The stem-cell niche as an entity of action. Nature. 2006;441(7097):1075-9. Review.

4. Morrison SJ, Spradling AC. Stem cells and niches: mechanisms that promote stem cell maintenance throughout life. Cell. 2008;132(4):598-611. Review.

5. Sheng XR, Posenau T, Gumulak-smith JJ, et al. Jak-STAT regulation of male germline stem cell establishment during Drosophila embryogenesis. Dev Biol. 2009;334(2):335-44.

6. Bausek N. JAK-STAT signaling in stem cells and their niches in Drosophila. JAKSTAT. 2013;2(3), e25686. Review.

7. Lin TY, Viswanathan S, Wood C, et al. Coordinate developmental control of the meiotic cell cycle and spermatid differentiation in Drosophila males. Development. 1996;122(4):1331-41.

8. Baker CC, Gim BS, Fuller MT. Cell type-specific translational repression of Cyclin B during meiosis in males. Development. 2015;142(19):3394-402.

9. Rhind N, Russell P. Signaling pathways that regulate cell division. Cold Spring Harb Perspect Biol. 2012;1:4(10).

10. Hochegger H, Takeda S, Hunt T. Cyclin-dependent kinases and cell-cycle transitions: does one fit all? Nat Rev Mol Cell Biol. 2008;9(11):910-6.

11. Kareta MS, Sage J, Wernig M. Crosstalk between stem cell and cell cycle machineries. Curr Opin Cell Biol. 2015;37:68-74.

12. Kotov A A, Olenkina O M, Kibanov M V et al. RNA helicase Belle (DDX3) is essential for male germline stem cell maintenance and division in Drosophila. Biochim Biophys Acta. 2016.

13. Cheng J, Türkel N, Hemati N, et al. Centrosome misorientation reduces stem cell division during ageing. Nature. 2008;456(7222): 599-604.

14. Yamashita YM, Jones DL, Fuller MT. Orientation of asymmetric stem cell division by the APC tumor suppressor and centrosome. Science. 2003;301(5639):1547-50.

15. Tran V, Feng L, Chen X. Asymmetric distribution of histones during Drosophila male germline stem cell asymmetric divisions. Chromosom Res. 2013;21(3):255-69. Review.

16. Inaba $M$, Venkei ZG, Yamashita YM. The polarity protein Baz forms a platform for the centrosome orientation during asymmetric stem cell division in the Drosophila male germline. Elife. 2015;20:4.
17. Wang Z, Lin H. The division of Drosophila germline stem cells and their precursors requires a specific cyclin. Curr Biol. 2005;15(4): 328-33.

18. Inaba M, Yuan H, Yamashita YM. String (Cdc25) regulates stem cell maintenance, proliferation and aging in Drosophila testis. Development. 2011;138(23):5079-86.

19.• Yamashita YM, Mahowald AP, Perlin JR, et al. Asymmetric inheritance of mother versus daughter centrosome in stem cell division. Science. 2007;315(5811):518-21. This study demonstrates that centrosomes are asymmetrically segregated between the GSC and differentiating GB.

20. Wodarz A, Ramrath A, Kuchinke U, et al. Bazooka provides an apical cue for Inscuteable localization in Drosophila neuroblasts. Nature. 1999;402(6761):544-7.

21. Benton R, St Johnston D. Drosophila PAR-1 and 14-3-3 inhibit Bazooka/PAR-3 to establish complementary cortical domains in polarized cells. Cell. 2003;115(6):691-704.

22. Yuan H, Chiang CY, Cheng J, et al. Regulation of cyclin A localization downstream of Par-1 function is critical for the centrosome orientation checkpoint in Drosophila male germline stem cells. Dev Biol. 2012;361(1):57-67.

23. Venkei ZG, Yamashita YM. The centrosome orientation checkpoint is germline stem cell specific and operates prior to the spindle assembly checkpoint in Drosophila testis. Development. 2015;142(1):62-9.

24. Tran V, Lim C, Xie J, Chen X. Asymmetric division of Drosophila male germline stem cell shows asymmetric histone distribution. Science. 2012;338(6107):679-82.

25.• Xie J, Wooten M, Tran V, et al. Histone H3 Threonine phosphorylation regulates asymmetric histone inheritance in the Drosophila male germline. Cell. 2015;163(4):920-33. This study demonstrates that a transient mitotic phosphorylation of preexisting histone H3 in GSCs allows for asymmetric segregation of chromatids based on histone age.

26. Panigada D, Grianti P, Nespoli A, et al. Yeast haspin kinase regulates polarity cues necessary for mitotic spindle positioning and is required to tolerate mitotic arrest. Dev Cell. 2013;26:483-95.

27. Wang F, Dai J, Daum JR, et al. Histone H3 Thr-3 phosphorylation by Haspin positions Aurora B at centromeres in mitosis. Science. 2010;330:231-5.

28. Dai J, Sultan S, Taylor SS, et al. The kinase haspin is required for mitotic histone $\mathrm{H} 3 \mathrm{Thr} 3$ phosphorylation and normal metaphase chromosome alignment. Genes Dev. 2005;19:472-88.

29. Yadlapalli S, Yamashita YM. Chromosome-specific nonrandom sister chromatid segregation during stem-cell division. Nature. 2013;498(7453):251-4. This study demonstrates that the $\mathbf{X}$ and $Y$ chromosome are non-randomly segregated in male GSCs. This is in contrast to autosomes which are randomly segregated in GSCs and differentiating GBs.

30. Li K, Kaufman TC. The homeotic target gene centrosomin encodes an essential centrosomal component. Cell. 1996;85(4):585-96.

31. Kracklauer MP, Banks SM, Xie X, et al. Drosophila klaroid encodes a SUN domain protein required for Klarsicht localization to the nuclear envelope and nuclear migration in the eye. Fly (Austin). 2007;1(2):75-85.

32. Mosley-Bishop KL, Li Q, Patterson L, et al. Molecular analysis of the klarsicht gene and its role in nuclear migration within differentiating cells of the Drosophila eye. Curr Biol. 1999;9(21):1211-20.

33. Phalke S, Nickel O, Walluscheck D, et al. Retrotransposon silencing and telomere integrity in somatic cells of Drosophila depends on the cytosine-5 methyltransferase DNMT2. Nat Genet. 2009;41(6):696-702.

34. Schwarz YB, Perrotta V. A new world of Polycombs: unexpected partnerships and emerging functions. Nat Rev Genet. 2013;14(12): 853-64. Review. 
35. Cao R, Zhang Y. The functions of E(Z)/EZH2-mediated methylation of lysine 27 in histone H3. Curr Opin Genet Dev. 2004;14(2): 155-64. Review.

36. Eun SH, Shi Z, Cui K, et al. A non-cell autonomous role of $\mathrm{E}(\mathrm{z})$ to prevent germ cells from turning on a somatic cell marker. Science. 2014;343(6178):1513-6.

37. Eissenberg JC, Lee MG, Schneider J, et al. The trithorax-group gene in Drosophila little imaginal discs encodes a trimethylated histone H3 Lys4 demethylase. Nat Struct Mol Biol. 2007;14(4): 344-6.

38. Sandstrom RS, Foret MR, Grow DA, et al. Epigenetic regulation by chromatin activation mark $\mathrm{H} 3 \mathrm{~K} 4 \mathrm{me} 3$ in primate progenitor cells within adult neurogenic niche. Sci Rep. 2014;4:5371.

39. Tarayrah L, Li Y, Gan Q, et al. Epigenetic regulator Lid maintains germline stem cells through regulating JAK-STAT signaling pathway activity. Biol Open. 2015;4(11):1518-27.

40. Tarayrah L, Herz HM, Shilatifard A, et al. Histone demethylase dUTX antagonizes JAK-STAT signaling to maintain proper gene expression and architecture of the Drosophila testis niche. Development. 2013;140(5):1014-23.

41. Björklund M, Taipale M, Varjosalo M, et al. Identification of pathways regulating cell size and cell-cycle progression by RNAi. Nature. 2006;439(7079):1009-13.

42. Müller P, Boutros M, Zeidler MP. Identification of JAK/STAT pathway regulators - insights from RNAi screens. Semin Cell Dev Biol. 2008;19(4):360-9. Review.

43. Harrison DA, McCoon PE, Binari R, et al. Drosophila unpaired encodes a secreted protein that activates the JAK signaling pathway. Genes Dev. 1998;12(20):3252-63.

44. Singh SR, Zheng Z, Wang H, et al. Competitiveness for the niche and mutual dependence of the germline and somatic stem cells in the Drosophila testis are regulated by the JAK/STAT signaling. J Cell Physiol. 2010;223(2):500-10.

45. Issigonis $\mathrm{M}$, Tulina $\mathrm{N}$, de Cuevas $\mathrm{M}$, et al. JAK-STAT signal inhibition regulates competition in the Drosophila testis stem cell niche. Science. 2009;326(5949):153-6.

46. Schulz C, Kiger AA, Tazuke SI, et al. A misexpression screen reveals effects of bag-of-marbles and TGF beta class signaling on the Drosophila male germ-line stem cell lineage. Genetics. 2004;167(2):707-23.

47. Shivdasani AA, Ingham PW. Regulation of stem cell maintenance and transit amplifying cell proliferation by tgf-beta signaling in Drosophila spermatogenesis. Curr Biol. 2003;13(23):2065-72.
48. Kawase E, Wong MD, Ding BC, et al. Gbb/Bmp signaling is essential for maintaining germline stem cells and for repressing bam transcription in the Drosophila testis. Development. 2004;131(6): 1365-75.

49. Inaba M, Buszczak M, Yamashita YM. Nanotubes mediate nichestem-cell signalling in the Drosophila testis. Nature. 2015;523(7560):329-32. This study is the first demonstration of a cilia-like structure in Drosophila stem cells potentially partaking in cellular signaling for stem cell maintenance.

50. Roth TM, Chiang CY, Inaba M, et al. Centrosome misorientation mediates slowing of the cell cycle under limited nutrient conditions in Drosophila male germline stem cells. Mol Biol Cell. 2012;23(8): 1524-32.

51. Chen H, Chen X, Zheng Y. The nuclear lamina regulates germline stem cell niche organization via modulation of EGFR signaling. Cell Stem Cell. 2013;13(1):73-86.

52. Amoyel M, Anderson J, Suisse A, et al. Socs36E controls niche competition by repressing MAPK signaling in the Drosophila testis. PLoS Genet. 2016;12(1), e1005815.

53. Michel M, Kupinski AP, Raabe I, et al. Hh signalling is essential for somatic stem cell maintenance in the Drosophila testis niche. Development. 2012;139(15):2663-9.

54. Brawley C, Matunis E. Regeneration of male germline stem cells by spermatogonial dedifferentiation in vivo. Science. 2004;304(5675): 1331-4.

55. Toledano H, D'Alterio C, Czech B, et al. The let-7-Imp axis regulates ageing of the Drosophila testis stem-cell niche. Nature. 2012;485(7400):605-10.

56. Siebold AP, Banerjee R, Tie F, et al. Polycomb Repressive Complex 2 and Trithorax modulate Drosophila longevity and stress resistance. Proc Natl Acad Sci U S A. 2010;107(1):169-74.

57. Li L, Greer C, Eisenman RN, et al. Essential functions of the histone demethylase lid. PLoS Genet. 2010;6(11), e1001221.

58. Greer EL, Maures TJ, Hauswirth AG, et al. Members of the H3K4 trimethylation complex regulate lifespan in a germline-dependent manner in C. elegans. Nature. 2010;466(7304):383-7.

59. Feser J, Truong D, Das C, et al. Elevated histone expression promotes life span extension. Mol Cell. 2010;39(5):724-35.

60. Watt FM, Driskell RR. The therapeutic potential of stem cells. Philos Trans R Soc Lond B Biol Sci. 2010;365(1537):155-63. 